\title{
The GGL Variational Principle for Constrained Mechanical Systems
}

\author{
P. L. Kinon, P. Betsch \\ Institute of Mechanics \\ Karlsruhe Institute of Technology (KIT) \\ Otto-Ammann-Platz 9, 76131 Karlsruhe, Germany \\ peter.betsch@kit.edu
}

\begin{abstract}
We present an extension of the Livens variational principle (sometimes also referred to as Hamilton-Pontryagin principle) to mechanical systems subject to holonomic constraints. The newly proposed principle embodies an index reduction in the spirit of the often-applied GGL stabilization and thus may be termed "GGL principle". The EulerLagrange equations of the GGL principle assume the form of differential-algebraic equations (DAEs) with differentiation index two. In contrast to the original GGLDAEs, the present formulation fits into the Hamiltonian framework of mechanics. Therefore, the GGL principle facilitates the design of symplectic integrators. In particular, it offers the possibility to construct variational integrators. This is illustrated with the development of a new first-order scheme which is symplectic by design. The numerical properties of the newly devised scheme are investigated in a representative example of a constrained mechanical system.
\end{abstract}

Keywords: Livens principle, Constrained dynamics, Gear-Gupta-Leimkuhler stabilization, Index reduction, Variational integrators.

\section{INTRODUCTION}

Dynamical systems may be formulated in various ways. The well-known Lagrangian and Hamiltonian formalisms both consider descriptive energetic scalars and deploy certain operations on them to generate the system's equations of motion. Another formulation, which unifies both abovementioned formalisms by means of independent position, velocity and momentum quantities has been proposed by Livens [1]. This Livens principle has been recently taken up by Bou-Rabee [2], Yoshimura \& Marsden [3] and Holm [4] under the name of Hamilton-Pontryagin principle due to its close relation to the Pontryagin principle from the field of optimal control [5]. Livens principle allows for an advantageous universal description due to its mixed character.

A large variety of dynamical systems are subject to constraints, which reduce the degrees of freedom of the system and impose some constraint function to be satisfied. When describing the system with redundant coordinates, the equations of motion emerge as a set of differential-algebraic equations (DAEs), which combine both differential equations and algebraic constraint equations. It is to mention that the numerical treatment of DAEs requires some additional effort compared to purely differential equations (cf. Kunkel \& Mehrmann [6]). As the constraints have to hold at every point in time (consistency condition), so-called secondary constraints for the position and velocity quantities are induced.

In a vast majority of dynamical problems, one cannot find an analytical solution. Thus, in recent times, the focus of scientific research has become to derive numerical integration methods, which are capable of solving the equations of motion approximately. Therefore, the class of structurepreserving integrators seeks to inherit the conservation principles of dynamical systems in a discrete sense (cf. monographs such as Hairer et al. [7] or Leimkuhler \& Reich [8]). The first contributions can be traced back to symplectic methods (see e.g. de Vogelaere [9]). In the field of mechanics, structure-preserving integration schemes can be mainly divided into two different groups: variational integrators and energy-momentum integrators. 
Variational integrators approximate the action integral and are typically able to conserve the symplectic structure as well as the system's momentum maps in a discrete sense (cf. Lew \& Mata [10]). These are consequences of the variational procedure of derivation (cf. Marsden \& West [11]). The main idea to find discrete counterparts of the variational principles goes back to Maeda [12]. Based on this concept, Marsden \& West [11] provided a framework of discrete Lagrangian and Hamiltonian mechanics. Until now, variational integrators have been developed for various applications, e.g. for constrained dynamical systems (see e.g. Leyendecker et al. [13]).

Integration schemes for constrained dynamics typically only account for the constraints on configuration level (primary constraint) but not for the secondary constraints and thus have a differentiation index of 3. This may lead to numerical instabilities, especially when singular points exist (cf. Yoshimura [14]). By replacing the primary constraints on position level with the secondary velocity-level constraints, DAEs with index 2 are obtained. Thus, the numerical problems can be avoided but violations of the primary constraints are induced (cf. Yoshimura [14]). However, this issue can be alleviated by extending the system of unknowns and coupling the secondary constraints into the equations. The most famous technique, the Gear-Gupta-Leimkuhler (GGL) stabilization, traces back to Gear et al. [15] in 1985 and is widely used until today. This classical GGL formulation relies on the direct modification of the equations of motion. Yet, this procedure leads to a destruction of the Hamiltonian structure such that most GGL stabilized integration schemes are not symplectic.

To the best of the author's knowledge, numerical integration schemes for constrained dynamics have been formulated either in a Hamiltonian or in a Lagrangian way. Moreover, variational integration schemes have not been yet constructed such that primary and secondary constraints are considered at once. Thus, this work tries to fill both gaps by introducing a novel Livens-based variational framework for the integration of dynamical systems accounting for both primary and secondary constraints. In particular, the new framework makes possible to justify the commonly used GGL formulation in a variational sense. Contrary to the original version, the newly proposed formalism provides index 2 DAEs with a Hamiltonian structure. Moreover, the novel variational principle opens up the possibility to develop new variational integration schemes with a GGL-type stabilization.

\section{FUNDAMENTALS}

\subsection{Livens principle}

Consider a dynamical system with $d$ degrees of freedom and positions $\boldsymbol{q} \in \mathbb{R}^{d}$. From Hamilton's principle of least action one can proceed by allowing the velocities to be independent variables $\boldsymbol{v} \in \mathbb{R}^{d}$. Thus, the kinematic relation $\dot{\boldsymbol{q}}=\boldsymbol{v}$ has to be enforced by means of a Lagrange multiplier $\boldsymbol{p} \in \mathbb{R}^{d}$. The corresponding augmented functional reads

$$
\tilde{S}(\boldsymbol{q}, \boldsymbol{v}, \boldsymbol{p})=\int_{0}^{T}[L(\boldsymbol{q}, \boldsymbol{v})+\boldsymbol{p} \cdot(\dot{\boldsymbol{q}}-\boldsymbol{v})] \mathrm{d} t,
$$

where $L(\boldsymbol{q}, \boldsymbol{v})$ is the Lagrangian. The functional (1) was firstly termed Livens principle (cf. Sect. 26.2 in Pars [16]) after G.H. Livens who proposed this functional for the first time (cf. Livens [1]). More recently, Marsden and co-workers [2,3] coined the name Hamilton-Pontryagin principle for this functional due to its close relation to the classical Pontryagin principle from the field of optimal control [5]. Due to its mixed character with three independent fields $(\boldsymbol{q}, \boldsymbol{v}, \boldsymbol{p})$, it resembles the $\mathrm{Hu}$-Washizu principle from the area of elasticity theory (cf. Washizu [17]). Livens principle unifies both Lagrangian and Hamiltonian viewpoints on mechanics and automatically accounts for the Legendre transformation.

By stating the stationarity condition $\delta \tilde{S}(\boldsymbol{q}, \boldsymbol{v}, \boldsymbol{p})=0$ and executing the variations with respect to 
every independent variable, one obtains the equations of motion in the form

$$
\begin{aligned}
& \dot{\boldsymbol{q}}=\boldsymbol{v}, \\
& \dot{\boldsymbol{p}}=\mathrm{D}_{1} L(\boldsymbol{q}, \boldsymbol{v}), \\
& \boldsymbol{p}=\mathrm{D}_{2} L(\boldsymbol{q}, \boldsymbol{v}) .
\end{aligned}
$$

With regard to (2c) the multiplier $p$ can be identified as the conjugate momentum, which thus directly emanates from the principle. Within the framework of Hamiltonian dynamics momentum variables have to be defined a priori or emerge from the Legendre transformation as a fiber derivative of $L(\boldsymbol{q}, \dot{\boldsymbol{q}})$. Note that after reinserting (2c) into (2b) and making use of (2a), Livens principle traces back to the Lagrangian equations of the second kind.

For natural mechanical systems the Lagrangian takes the form

$$
L(\boldsymbol{q}, \boldsymbol{v})=\frac{1}{2} \boldsymbol{v} \cdot \boldsymbol{M} \boldsymbol{v}-V(\boldsymbol{q})
$$

where $\boldsymbol{M}$ is the mass matrix and $V(\boldsymbol{q})$ is a potential function. Now (2c) yields $\boldsymbol{p}=\boldsymbol{M} \boldsymbol{v}$, so that (2a) and (2b) can be rewritten as

$$
\begin{aligned}
& \dot{\boldsymbol{q}}=\boldsymbol{M}^{-1} \boldsymbol{p}, \\
& \dot{\boldsymbol{p}}=-\mathrm{D} V(\boldsymbol{q}) .
\end{aligned}
$$

These equations correspond to the Hamiltonian form of the equations of motion. Making use of the phase space vector $z=\left[\boldsymbol{q}^{\mathrm{T}}, \boldsymbol{p}^{\mathrm{T}}\right]^{\mathrm{T}}$ and the symplectic structure matrix

$$
\mathbb{J}=\left[\begin{array}{cc}
\mathbf{0}_{d \times d} & \boldsymbol{I}_{d \times d} \\
-\boldsymbol{I}_{d \times d} & \mathbf{0}_{d \times d}
\end{array}\right],
$$

where $\boldsymbol{I}_{d \times d} \in \mathbb{R}^{d \times d}$ denotes the $d \times d$ identity matrix, the Hamiltonian equations of motion read

$$
\dot{\boldsymbol{z}}=\mathbb{J} \mathrm{D} H(\boldsymbol{z})
$$

where the standard Hamiltonian function corresponding to (3) is given by

$$
H=\frac{1}{2} \boldsymbol{p} \cdot \boldsymbol{M}^{-1} \boldsymbol{p}+V(\boldsymbol{q})
$$

\subsection{Symplectic structure of dynamics}

Let us firstly introduce a bilinear and skew-symmetric function $\Omega: \mathbb{R}^{2 d} \times \mathbb{R}^{2 d} \rightarrow \mathbb{R}$ acting on two elements $\boldsymbol{\xi}, \boldsymbol{\eta} \in \mathbb{R}^{2 d}$ which assume an ordering of components as in the phase space vector, such that exemplarily

$$
\boldsymbol{\xi}=\left[\begin{array}{llllllll}
\xi_{1}^{(1)} & \xi_{1}^{(2)} & \ldots & \xi_{1}^{(d)} & \xi_{2}^{(1)} & \xi_{2}^{(2)} & \ldots & \xi_{2}^{(d)}
\end{array}\right]^{\mathrm{T}}
$$

Bilinearity refers to the fact that $\Omega$ is linear in both arguments. Skew-symmetry implies that $\Omega(\boldsymbol{\xi}, \boldsymbol{\eta})=-\Omega(\boldsymbol{\eta}, \boldsymbol{\xi})$. The canonical structure matrix introduced in (5) gives rise to the symplectic two-form

$$
\Omega(\boldsymbol{\xi}, \boldsymbol{\eta})=\boldsymbol{\xi} \cdot \mathbb{J}^{-1} \boldsymbol{\eta}
$$

A map $\Psi: \mathbb{R}^{2 \mathrm{~d}} \rightarrow \mathbb{R}^{2 \mathrm{~d}}$ is called symplectic if it leaves the symplectic two-form $\Omega$ invariant in the sense that

$$
\Omega(\mathrm{D} \Psi(z) \boldsymbol{\xi}, \mathrm{D} \Psi(z) \boldsymbol{\eta})=\Omega(\boldsymbol{\xi}, \boldsymbol{\eta})
$$




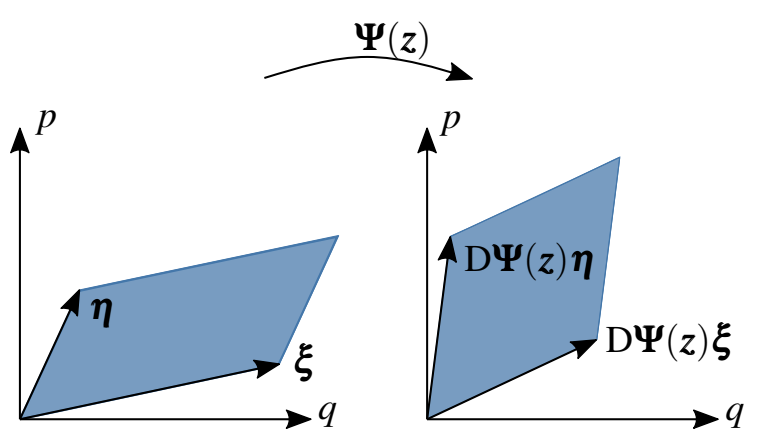

Figure 1. Symplectic area preservation for $d=1$ (inspired by Hairer et al. [7])

where the original two-form is equal to the two-form of the transports of $\boldsymbol{\xi}$ and $\boldsymbol{\eta}$ under the linearization of $\boldsymbol{\Psi}$. Fig. 1 displays the mapping of elements $\boldsymbol{\xi}$ and $\boldsymbol{\eta}$ by $\boldsymbol{\Psi}$ and the preservation of the symplectic two-form, representing the oriented area for $d=1$. Making use of the definition (9), the last equation leads to a symplecticness condition that reads

$$
\mathrm{D} \Psi(z)^{\mathrm{T}} \mathbb{J}^{-1} \mathrm{D} \Psi(z)=\mathbb{J}^{-1} .
$$

The wedge product of two differential one-forms $\mathrm{d} \boldsymbol{a} \in \mathbb{R}^{d}$ and $\mathrm{d} \boldsymbol{b} \in \mathbb{R}^{d}$ acting on any two vectors $\boldsymbol{\xi}, \boldsymbol{\eta} \in \mathbb{R}^{2 d}$ is given by (cf. Leimkuhler \& Reich [8])

$$
(\mathrm{d} \boldsymbol{a} \wedge \mathrm{d} \boldsymbol{b})(\boldsymbol{\xi}, \boldsymbol{\eta})=\sum_{i=1}^{d}\left(\mathrm{~d} a_{i} \wedge \mathrm{d} b_{i}\right)(\boldsymbol{\xi}, \boldsymbol{\eta})=\sum_{i=1}^{d}\left(\mathrm{~d} b_{i}(\boldsymbol{\xi}) \mathrm{d} a_{i}(\boldsymbol{\eta})-\mathrm{d} a_{i}(\boldsymbol{\xi}) \mathrm{d} b_{i}(\boldsymbol{\eta})\right) .
$$

Thus, the symplectic two form (9) can be rewritten in terms of the wedge product as

$$
\Omega(\boldsymbol{\xi}, \boldsymbol{\eta})=\sum_{i=1}^{d} \mathrm{~d} q_{i} \wedge \mathrm{d} p_{i}(\boldsymbol{\xi}, \boldsymbol{\eta}),
$$

where the differential one-forms $\mathrm{d} q_{i}, \mathrm{~d} p_{i}$ extract the $i$-th coordinate or momentum component, respectively, such that

$$
\mathrm{d} q_{i}(\boldsymbol{\xi})=\xi_{1}^{(i)}, \quad \mathrm{d} p_{i}(\boldsymbol{\xi})=\xi_{2}^{(i)} .
$$

Omitting the arguments, the symplectic two-form can be rewritten more briefly in vector notation as

$$
\Omega=\mathrm{d} \boldsymbol{q} \wedge \mathrm{d} \boldsymbol{p} .
$$

Note that this representation is only a briefer notation of (13) that still accounts for the summation of the wedge product of scalar one-forms. One can show that Hamiltonian flow maps are symplectic. Thus, it is equivalent to say that the symplectic two-form (15) is conserved along solutions of the Hamiltonian equations of motions (6), viz.

$$
\frac{\mathrm{d}}{\mathrm{d} t} \Omega=\frac{\mathrm{d}}{\mathrm{d} t}(\mathrm{~d} \boldsymbol{q} \wedge \mathrm{d} \boldsymbol{p})=0 .
$$

For differential one-forms, given in vector notation as $\mathrm{d} \boldsymbol{a}, \mathrm{d} \boldsymbol{b}$ and $\mathrm{d} \boldsymbol{c} \in \mathbb{R}^{d}$, any scalar valued quantities $\alpha, \beta \in \mathbb{R}$, any matrix $\boldsymbol{A} \in \mathbb{R}^{d \times d}$ and any symmetric matrix $\boldsymbol{B}=\boldsymbol{B}^{\mathrm{T}} \in \mathbb{R}^{d \times d}$ the wedge product defined in (12) has the following properties (see, for example, Leimkuhler \& Reich [8]):

$$
\begin{aligned}
\mathrm{d} \boldsymbol{a} \wedge \mathrm{d} \boldsymbol{b} & =-\mathrm{d} \boldsymbol{b} \wedge \mathrm{d} \boldsymbol{a}, \\
\mathrm{d} \boldsymbol{a} \wedge(\alpha \mathrm{d} \boldsymbol{b}+\beta \mathrm{d} \boldsymbol{c}) & =\alpha \mathrm{d} \boldsymbol{a} \wedge \mathrm{d} \boldsymbol{b}+\beta \mathrm{d} \boldsymbol{a} \wedge \mathrm{d} \boldsymbol{c}, \\
\mathrm{d} \boldsymbol{a} \wedge(\boldsymbol{A} \mathrm{d} \boldsymbol{b}) & =\left(\boldsymbol{A}^{\mathrm{T}} \mathrm{d} \boldsymbol{a}\right) \wedge \mathrm{d} \boldsymbol{b}, \\
\mathrm{d} \boldsymbol{a} \wedge(\boldsymbol{B} \mathrm{d} \boldsymbol{a}) & =0 .
\end{aligned}
$$




\subsection{GGL method for constrained mechanical systems}

Assume that the coordinates $\boldsymbol{q}$ are redundant due to the presence of $m$ independent scleronomic, holonomic constraints $g_{k}: \mathbb{R}^{d} \rightarrow \mathbb{R}(k=1, \ldots, m)$. The constraints can be comprised in a column vector $g \in \mathbb{R}^{m}$, such that

$$
\boldsymbol{g}(\boldsymbol{q})=\mathbf{0} .
$$

Since all constraint functions shall be independent, the constraint Jacobian $\boldsymbol{G}(\boldsymbol{q})=\mathrm{D} \boldsymbol{g}(\boldsymbol{q})$ is of rank $m$. As (18) is true for any point in time, the time derivative has to vanish accordingly (consistency condition). Thus, the constraints on velocity level or secondary constraints

$$
\frac{\mathrm{d}}{\mathrm{d} t} \boldsymbol{g}(\boldsymbol{q})=\boldsymbol{G}(\boldsymbol{q}) \dot{\boldsymbol{q}}=\mathbf{0}
$$

are induced. It is well-known that the motion of the constrained mechanical systems under consideration is governed by differential-algebraic equations (DAEs) which have differentiation index $v=3$. These equations of motion can be derived with a variational approach, which augments Livens principle (1). Accordingly, introducing $\hat{S}(\boldsymbol{q}, \boldsymbol{v}, \boldsymbol{p}, \boldsymbol{\lambda})=\tilde{S}(\boldsymbol{q}, \boldsymbol{v}, \boldsymbol{p})+\int_{0}^{T} \boldsymbol{\lambda} \cdot \boldsymbol{g}(\boldsymbol{q}) \mathrm{d} t$, stating the stationary condition $\delta \hat{S}(\boldsymbol{q}, \boldsymbol{v}, \boldsymbol{p}, \boldsymbol{\lambda})=0$ and eliminating the velocities as above leads to an extension of the Hamiltonian equations (4) for constrained systems, such that the index-3 DAEs are obtained as

$$
\begin{aligned}
\dot{\boldsymbol{q}} & =\boldsymbol{M}^{-1} \boldsymbol{p}, \\
\dot{\boldsymbol{p}} & =-\mathrm{D} V(\boldsymbol{q})-\boldsymbol{G}(\boldsymbol{q})^{\mathrm{T}} \boldsymbol{\lambda}, \\
\mathbf{0} & =\boldsymbol{g}(\boldsymbol{q}) .
\end{aligned}
$$

The classical GGL stabilization, which traces back to Gear et al. [15], represents an index reduction technique by minimal extension (see Kunkel \& Mehrmann [6]). The main idea of the GGL stabilization is to couple the secondary constraints into the dynamics by making use of additional variables $\boldsymbol{\gamma} \in \mathbb{R}^{m}$, such that the system of equations of motion is extended and the differentiation index drops to $v=2$. Correspondingly, the numerical ill-conditioning of index-3 DAEs are alleviated without having the drawback of drift phenomena. The resulting index-2 DAEs can be written in the form

$$
\begin{aligned}
\dot{\boldsymbol{q}} & =\boldsymbol{M}^{-1} \boldsymbol{p}+\boldsymbol{G}(\boldsymbol{q})^{\mathrm{T}} \boldsymbol{\gamma}, \\
\dot{\boldsymbol{p}} & =-\mathrm{D} V(\boldsymbol{q})-\boldsymbol{G}(\boldsymbol{q})^{\mathrm{T}} \boldsymbol{\lambda}, \\
\mathbf{0} & =\boldsymbol{g}(\boldsymbol{q}), \\
\mathbf{0} & =\boldsymbol{G}(\boldsymbol{q}) \boldsymbol{M}^{-1} \boldsymbol{p} .
\end{aligned}
$$

Ever since, the GGL stabilization has been widely used and is thus of great importance. Numerical methods can be constructed directly by discretizing the DAEs (21). Note, however, that due to the GGL modification of the kinematic equation (21a), the system (21) loses its Hamiltonian structure. For the time-continuous case, some algebra leads to $\boldsymbol{\gamma}=\mathbf{0}$. Consequently, the GGL-DAEs boil down to the standard formulation (20).

\section{GGL PRINCIPLE}

\subsection{Governing equations}

The newly proposed GGL principle relies on a generalization of Livens principle (1) by considering Lagrange multipliers $\boldsymbol{\lambda}, \boldsymbol{\gamma} \in \mathbb{R}^{m}$ to enforce the primary constraints (18) and secondary constraints (19), respectively. Imposing stationarity on a corresponding augmented action integral

$$
\delta S_{\mathrm{GGL}}(\boldsymbol{q}, \boldsymbol{v}, \boldsymbol{p}, \boldsymbol{\lambda}, \boldsymbol{\gamma})=0
$$


with

$$
S_{\mathrm{GGL}}=\int_{0}^{T}\left[L(\boldsymbol{q}, \boldsymbol{v})-\boldsymbol{\lambda} \cdot \boldsymbol{g}(\boldsymbol{q})+\boldsymbol{p} \cdot\left(\dot{\boldsymbol{q}}-\boldsymbol{v}-\boldsymbol{M}^{-1} \boldsymbol{G}(\boldsymbol{q})^{\mathrm{T}} \boldsymbol{\gamma}\right)\right] \mathrm{d} t,
$$

yields the stationarity conditions

$$
\begin{array}{r}
\left.\int_{0}^{T} \delta \boldsymbol{p} \cdot\left(\dot{\boldsymbol{q}}-\boldsymbol{v}-\boldsymbol{M}^{-1} \boldsymbol{G}(\boldsymbol{q})^{\mathrm{T}} \boldsymbol{\gamma}\right)\right) \mathrm{d} t=0, \\
\int_{0}^{T}\left(\mathrm{D}_{1} L(\boldsymbol{q}, \boldsymbol{v}) \cdot \delta \boldsymbol{q}-\boldsymbol{G}(\boldsymbol{q})^{\mathrm{T}} \boldsymbol{\lambda} \cdot \delta \boldsymbol{q}+\boldsymbol{p} \cdot \delta \dot{\boldsymbol{q}}-\boldsymbol{p} \cdot \boldsymbol{M}^{-1} \delta \boldsymbol{G}(\boldsymbol{q})^{\mathrm{T}} \boldsymbol{\gamma}\right) \mathrm{d} t=0, \\
\int_{0}^{T} \delta \boldsymbol{v} \cdot\left(\mathrm{D}_{2} L(\boldsymbol{q}, \boldsymbol{v})-\boldsymbol{p}\right) \mathrm{d} t=0, \\
\int_{0}^{T} \delta \boldsymbol{\lambda} \cdot \boldsymbol{g}(\boldsymbol{q}) \mathrm{d} t=0, \\
\int_{0}^{T} \boldsymbol{p} \cdot \boldsymbol{M}^{-1} \boldsymbol{G}(\boldsymbol{q})^{\mathrm{T}} \boldsymbol{\delta} \boldsymbol{\gamma} \mathrm{d} t=0 .
\end{array}
$$

As one can see, relation (24b) requires some more effort in order to achieve the final EulerLangrange equation. Therefore, $\delta \dot{\boldsymbol{q}}$ can be replaced through integration by parts such that

$$
\int_{0}^{T} \boldsymbol{p} \cdot \delta \dot{\boldsymbol{q}} \mathrm{d} t=-\int_{0}^{T} \delta \boldsymbol{q} \cdot \dot{\boldsymbol{p}} \mathrm{d} t+\delta \boldsymbol{q}(T) \cdot \boldsymbol{p}(T)-\delta \boldsymbol{q}(0) \cdot \boldsymbol{p}(0) .
$$

The endpoint conditions on admissible variations $\delta \boldsymbol{q}(0)=\delta \boldsymbol{q}(T)=0$ make the latter two terms vanish. Furthermore, the variation of the gradient of the constraint functions can be executed as

$$
\delta \boldsymbol{G}(\boldsymbol{q})=\mathrm{D} \boldsymbol{G}(\boldsymbol{q}) \boldsymbol{\delta} \boldsymbol{q}=\mathrm{D}^{2} \boldsymbol{g}(\boldsymbol{q}) \delta \boldsymbol{q} .
$$

In order to avoid the third order expression $\mathrm{D}^{2} \boldsymbol{g}(\boldsymbol{q})$, the stationary condition (24b) can be written in terms of the individual constraint functions $g_{\mathrm{k}}(\boldsymbol{q})$ for $k=1, \ldots, m$. Thus, the variation of the constraint gradients is given by

$$
\delta\left(\mathrm{D} g_{k}(\boldsymbol{q})\right)=\mathrm{D}^{2} g_{k}(\boldsymbol{q}) \boldsymbol{\delta} \boldsymbol{q},
$$

where the constraint Hessian $\mathrm{D}^{2} g_{k}(\boldsymbol{q}) \in \mathbb{R}^{d \times d}$. Consequently, the arbitrariness of the variations $\delta \boldsymbol{q}, \delta \boldsymbol{v}, \delta \boldsymbol{p}, \delta \boldsymbol{\lambda}$ and $\delta \boldsymbol{\gamma}$ can be taken into account such that the governing DAEs are deduced as

$$
\begin{aligned}
\dot{\boldsymbol{q}} & =\boldsymbol{v}+\boldsymbol{M}^{-1} \boldsymbol{G}(\boldsymbol{q})^{\mathrm{T}} \boldsymbol{\gamma}, \\
\dot{\boldsymbol{p}} & =\mathrm{D}_{1} L(\boldsymbol{q}, \boldsymbol{v})-\boldsymbol{G}(\boldsymbol{q})^{\mathrm{T}} \boldsymbol{\lambda}-\sum_{k=1}^{m} \gamma_{k} \mathrm{D}^{2} g_{k}(\boldsymbol{q}) \boldsymbol{M}^{-1} \boldsymbol{p}, \\
\boldsymbol{p} & =\mathrm{D}_{2} L(\boldsymbol{q}, \boldsymbol{v}) \\
\mathbf{0} & =\boldsymbol{g}(\boldsymbol{q}), \\
\mathbf{0} & =\boldsymbol{G}(\boldsymbol{q}) \boldsymbol{M}^{-1} \boldsymbol{p} .
\end{aligned}
$$

Thus equations in the fashion of the standard GGL stabilization (21) are obtained with an additional term in the momentum equation. Note that $(28 \mathrm{c})$ represents the fiber derivative of the newly proposed variational principle, such that the Lagrange multiplier $\boldsymbol{p}$ denotes the conjugate momenta. By introducing the secondary constraints to the functional, the resulting Euler-Lagrange equations are DAEs with index $v=2$, similarly to the GGL stabilized equations of motion, whereas the standard DAEs of constrained dynamics have index $v=3$. Thus, the newly established DAEs (28) can be regarded as an extension to the classical GGL stabilization. Similar to the classical GGL stabilization one obtains $\boldsymbol{\gamma}=\mathbf{0}$ for the time-continuous case. However, the third term on the right-hand side of (28b) is of crucial importance as it maintains the Hamiltonian structure of the equations of motion, in contrast to the classical GGL method. 


\subsection{Hamiltonian structure}

The equations of motion induced by the GGL principle have Hamiltonian structure, viz.

$$
\begin{aligned}
& \dot{\boldsymbol{q}}=+\mathrm{D}_{2} H_{\mathrm{GGL}}(\boldsymbol{q}, \boldsymbol{p}, \boldsymbol{\lambda}, \boldsymbol{\gamma}), \\
& \dot{\boldsymbol{p}}=-\mathrm{D}_{1} H_{\mathrm{GGL}}(\boldsymbol{q}, \boldsymbol{p}, \boldsymbol{\lambda}, \boldsymbol{\gamma}), \\
& \mathbf{0}=+\mathrm{D}_{3} H_{\mathrm{GGL}}(\boldsymbol{q}, \boldsymbol{p}, \boldsymbol{\lambda}, \boldsymbol{\gamma}), \\
& \mathbf{0}=+\mathrm{D}_{4} H_{\mathrm{GGL}}(\boldsymbol{q}, \boldsymbol{p}, \boldsymbol{\lambda}, \boldsymbol{\gamma}),
\end{aligned}
$$

with a corresponding augmented Hamiltonian

$$
H_{\mathrm{GGL}}(\boldsymbol{q}, \boldsymbol{p}, \boldsymbol{\lambda}, \boldsymbol{\gamma})=\frac{1}{2} \boldsymbol{p} \cdot \boldsymbol{M}^{-1} \boldsymbol{p}+V(\boldsymbol{q})+\boldsymbol{\lambda} \cdot \boldsymbol{g}(\boldsymbol{q})+\boldsymbol{\gamma} \cdot \boldsymbol{G}(\boldsymbol{q}) \boldsymbol{M}^{-1} \boldsymbol{p} .
$$

The above equations can be related to the Euler-Lagrange equations (28) of the GGL functional after elimination of the velocities by employing the Legendre transformation (28c). Next, we show that the equations of motion of the novel framework conserve the Hamiltonian exactly. For that purpose, we compute the time derivative of the augmented Hamiltonian (30) such that

$$
\begin{aligned}
\frac{\mathrm{d}}{\mathrm{d} t} H_{\mathrm{GGL}} & =\mathrm{D}_{1} H_{\mathrm{GGL}} \cdot \dot{\boldsymbol{q}}+\mathrm{D}_{2} H_{\mathrm{GGL}} \cdot \dot{\boldsymbol{p}}+\mathrm{D}_{3} H_{\mathrm{GGL}} \cdot \dot{\boldsymbol{\lambda}}+\mathrm{D}_{4} H_{\mathrm{GGL}} \cdot \dot{\boldsymbol{\gamma}} \\
& =\mathrm{D}_{1} H_{\mathrm{GGL}} \cdot \mathrm{D}_{2} H_{\mathrm{GGL}}-\mathrm{D}_{2} H_{\mathrm{GGL}} \cdot \mathrm{D}_{1} H_{\mathrm{GGL}}+\mathbf{0} \cdot \dot{\boldsymbol{\lambda}}+\mathbf{0} \cdot \dot{\boldsymbol{\gamma}}=0,
\end{aligned}
$$

where the Hamiltonian equations of motion (29) have been considered. As the augmented Hamiltonian (30) is conserved along solutions of the equations of motion, also the Hamiltonian $H$ itself is conserved since both constraints on configuration level and momentum level are identically zero such that $\dot{H}=0$. In contrast to the originial GGL formulation (cf. Sect. 2.3), this conservation law holds regardless of the actual value of the Lagrange multipliers $\boldsymbol{\gamma}$.

\subsection{Symplectic structure}

We show that the GGL functional inherits the symplectic structure of Hamiltonian systems and thus the symplectic two-form is conserved along solutions of (28) or (29), respectively. We begin by deriving the total differentials based on the equations of motion as

$$
\begin{aligned}
\mathrm{d} \dot{\boldsymbol{q}} & =\mathrm{D}_{21}^{2} H(\boldsymbol{q}, \boldsymbol{p}) \mathrm{d} \boldsymbol{q}+\mathrm{D}_{22}^{2} H(\boldsymbol{q}, \boldsymbol{p}) \mathrm{d} \boldsymbol{p}+\mathrm{d}\left(\mathrm{D}_{2} \boldsymbol{g}^{\mathbf{v}}(\boldsymbol{q}, \boldsymbol{p})^{\mathrm{T}} \boldsymbol{\gamma}\right), \\
\mathrm{d} \dot{\boldsymbol{p}} & =-\mathrm{D}_{11}^{2} H(\boldsymbol{q}, \boldsymbol{p}) \mathrm{d} \boldsymbol{q}-\mathrm{D}_{12}^{2} H(\boldsymbol{q}, \boldsymbol{p}) \mathrm{d} \boldsymbol{p}-\mathrm{d}\left(\mathrm{D}^{\mathbf{q}}(\boldsymbol{q})^{\mathrm{T}} \boldsymbol{\lambda}\right)-\mathrm{d}\left(\mathrm{D}_{1} \boldsymbol{g}^{\mathbf{v}}(\boldsymbol{q}, \boldsymbol{p})^{\mathrm{T}} \boldsymbol{\gamma}\right), \\
\mathbf{0} & =\mathrm{D}^{\mathbf{q}}(\boldsymbol{q}) \mathrm{d} \boldsymbol{q}, \\
\mathbf{0} & =\mathrm{D}_{1} \boldsymbol{g}^{\mathbf{v}}(\boldsymbol{q}, \boldsymbol{p}) \mathrm{d} \boldsymbol{q}+\mathrm{D}_{2} \boldsymbol{g}^{\mathbf{v}}(\boldsymbol{q}, \boldsymbol{p}) \mathrm{d} \boldsymbol{p},
\end{aligned}
$$

where we have introduced the distinct functions $\boldsymbol{g}^{\mathbf{q}}(\boldsymbol{q})=\boldsymbol{g}(\boldsymbol{q})$ for the holonomic constraint on configuration level and $\boldsymbol{g}^{\mathbf{v}}(\boldsymbol{q}, \boldsymbol{p})=\mathrm{D} \boldsymbol{g}(\boldsymbol{q}) \boldsymbol{M}^{-1} \boldsymbol{p}$ for the corresponding constraint on momentum level. It is straightforward to compute the temporal evolution of $\Omega$ by means of the product rule such that

$$
\frac{\mathrm{d}}{\mathrm{d} t} \Omega=\mathrm{d} \dot{\boldsymbol{q}} \wedge \mathrm{d} \boldsymbol{p}+\mathrm{d} \boldsymbol{q} \wedge \mathrm{d} \dot{\boldsymbol{p}},
$$

into which the above differential equations (32a) and (32b) can be inserted. One can consider the symmetry of the Hessian of $H$ and make use of the properties of the wedge product. Note that $\mathrm{D}_{11}^{2} H$ and $\mathrm{D}_{22}^{2} H$ both are symmetric matrices, such that

$$
\begin{aligned}
\mathrm{D}_{22}^{2} H(\boldsymbol{q}, \boldsymbol{p}) \mathrm{d} \boldsymbol{p} \wedge \mathrm{d} \boldsymbol{p} & =0, \\
-\mathrm{d} \boldsymbol{q} \wedge \mathrm{D}_{11}^{2} H(\boldsymbol{q}, \boldsymbol{p}) \mathrm{d} \boldsymbol{q} & =0,
\end{aligned}
$$


due to property (17d). Moreover, the two terms with the off-diagonal entries of the Hessian of $H$ cancel each other out, because

$$
\mathrm{D}_{21}^{2} H(\boldsymbol{q}, \boldsymbol{p}) \mathrm{d} \boldsymbol{q} \wedge \mathrm{d} \boldsymbol{p}=\left(\mathrm{D}_{12}^{2} H(\boldsymbol{q}, \boldsymbol{p})\right)^{\mathrm{T}} \mathrm{d} \boldsymbol{q} \wedge \mathrm{d} \boldsymbol{p}=\mathrm{d} \boldsymbol{q} \wedge \mathrm{D}_{12}^{2} H(\boldsymbol{q}, \boldsymbol{p}) \mathrm{d} \boldsymbol{p},
$$

where in the last equation property $(17 \mathrm{c})$ has been used. The term stemming from the primary constraints can be written as

$$
\mathrm{d}\left(\mathrm{D}^{\mathbf{q}}(\boldsymbol{q})^{\mathrm{T}} \boldsymbol{\lambda}\right) \wedge \mathrm{d} \boldsymbol{q}=\mathrm{D}^{\mathbf{q}}(\boldsymbol{q})^{\mathrm{T}} \mathrm{d} \boldsymbol{\lambda} \wedge \mathrm{d} \boldsymbol{q}+\sum_{\mathrm{k}=1}^{\mathrm{m}} \lambda_{\mathrm{k}} \mathrm{D}^{2} g_{\mathrm{k}}(\boldsymbol{q}) \mathrm{d} \boldsymbol{q} \wedge \mathrm{d} \boldsymbol{q}=\mathrm{d} \boldsymbol{\lambda} \wedge \mathrm{D} \boldsymbol{g}^{\mathbf{q}}(\boldsymbol{q}) \mathrm{d} \boldsymbol{q}=0,
$$

where again (17c) and (17d) have been used along with (32c). Therefore, all terms emerging from the right-hand side of (33) cancel out except for those containing the constraint on momentum level $\boldsymbol{g}^{\mathbf{v}}(\boldsymbol{q}, \boldsymbol{p})$. We therefore obtain

$$
\begin{aligned}
\frac{\mathrm{d}}{\mathrm{d} t} \Omega & =\mathrm{d}\left(\mathrm{D}_{2} \boldsymbol{g}^{\mathbf{v}}(\boldsymbol{q}, \boldsymbol{p})^{\mathrm{T}} \boldsymbol{\gamma}\right) \wedge \mathrm{d} \boldsymbol{p}-\mathrm{d} \boldsymbol{q} \wedge \mathrm{d}\left(\mathrm{D}_{1} \boldsymbol{g}^{\mathbf{v}}(\boldsymbol{q}, \boldsymbol{p})^{\mathrm{T}} \boldsymbol{\gamma}\right) \\
& =\mathrm{D}_{2} \boldsymbol{g}^{\mathbf{v} \mathrm{T}} \mathrm{d} \boldsymbol{\gamma} \wedge \mathrm{d} \boldsymbol{p}+\mathrm{D}_{1} \boldsymbol{g}^{\mathbf{v} \mathrm{T}} \mathrm{d} \boldsymbol{\gamma} \wedge \mathrm{d} \boldsymbol{q}+\sum_{k=1}^{m} \gamma_{k} \mathrm{D}_{12}^{2} g_{k}^{\mathbf{v}} \mathrm{d} \boldsymbol{q} \wedge \mathrm{d} \boldsymbol{p}+\sum_{k=1}^{m} \gamma_{k} \mathrm{D}_{21}^{2} g_{k}^{\mathbf{v}} \mathrm{d} \boldsymbol{p} \wedge \mathrm{d} \boldsymbol{q} \\
& =\mathrm{d} \boldsymbol{\gamma} \wedge\left(\mathrm{D}_{1} \boldsymbol{g}^{\mathbf{v}}(\boldsymbol{q}, \boldsymbol{p}) \mathrm{d} \boldsymbol{q}+\mathrm{D}_{2} \boldsymbol{g}^{\mathbf{v}}(\boldsymbol{q}, \boldsymbol{p}) \mathrm{d} \boldsymbol{p}\right)+\sum_{k=1}^{m} \gamma_{k}\left(\mathrm{D}_{12}^{2} g_{k}^{\mathbf{v}}-\left(\mathrm{D}_{21}^{2} g_{k}^{\mathbf{v}}\right)^{\mathrm{T}}\right) \mathrm{d} \boldsymbol{q} \wedge \mathrm{d} \boldsymbol{p}=0,
\end{aligned}
$$

where the first term vanishes in view of the total differential of the secondary constraint (32d) and the second one cancels due to the symmetry of the Hessian of $g_{k}^{\mathbf{v}}$. Note that it has been taken into account that terms including $\mathrm{D}_{11}^{2} g_{k}^{\mathbf{v}}$ and $\mathrm{D}_{22}^{2} g_{k}^{\mathbf{v}}$, respectively, cancel due to their symmetry. This proofs the symplecticness of the equations of motion emanating from the GGL functional. Again this property does not depend on the Lagrange multiplier $\gamma$, which is an advantage over the original GGL method by Gear et al. [15], for which $\boldsymbol{\gamma}=\mathbf{0}$ is required in order to conserve $\Omega$.

\section{GGL VARIATIONAL INTEGRATOR}

We next illustrate how the GGL principle introduced in Section 3 can be employed to derive a variational integrator. Subsequently, structure-preserving properties of the newly devised variational integrator will be considered.

\subsection{Governing Equations}

Let us construct a time-stepping scheme by means of a direct discretization of the GGL functional (23). Enforcing the constraint on configuration level in the endpoint and the constraint on momentum level in an intermediate state and discretizing the velocity by means of an explicit Euler method, we obtain the discrete action integral

$$
S_{\mathrm{d}}=\sum_{n=0}^{N-1}\left[h L\left(\boldsymbol{q}^{n}, \boldsymbol{v}^{n}\right)-h \boldsymbol{\lambda}^{n+1} \cdot \boldsymbol{g}\left(\boldsymbol{q}^{n+1}\right)+\boldsymbol{p}^{n+1} \cdot\left(\boldsymbol{q}^{n+1}-\boldsymbol{q}^{n}-h \boldsymbol{v}^{n}-h \boldsymbol{M}^{-1} \boldsymbol{G}(\overline{\boldsymbol{q}})^{\mathrm{T}} \boldsymbol{\gamma}^{n+1}\right)\right],
$$

where the configuration variable $\overline{\boldsymbol{q}}=\boldsymbol{q}^{n}+h \boldsymbol{v}^{n}$ has been introduced. Stationarity conditions can be applied directly to the discrete functional, yielding

$$
\begin{aligned}
& \sum_{n=0}^{N-1} \delta \boldsymbol{p}^{n+1} \cdot\left(\boldsymbol{q}^{n+1}-\boldsymbol{q}^{n}-h \boldsymbol{v}^{n}-h \boldsymbol{M}^{-1} \boldsymbol{G}(\overline{\boldsymbol{q}})^{\mathrm{T}} \boldsymbol{\gamma}^{n+1}\right)=0, \\
& \sum_{n=0}^{N-1} \delta \boldsymbol{q}^{n} \cdot\left(h \mathrm{D}_{1} L\left(\boldsymbol{q}^{n}, \boldsymbol{v}^{n}\right)-\boldsymbol{p}^{n+1}-h \sum_{k=1}^{m} \gamma_{k}^{n+1} \mathrm{D}^{2} g_{k}(\overline{\boldsymbol{q}}) \boldsymbol{M}^{-1} \boldsymbol{p}^{n+1}\right) \\
& +\sum_{n=0}^{N-1} \delta \boldsymbol{q}^{n+1} \cdot\left(-h \boldsymbol{G}\left(\boldsymbol{q}^{n+1}\right)^{\mathrm{T}} \boldsymbol{\lambda}^{n+1}+\boldsymbol{p}^{n+1}\right)=0 \\
& \sum_{n=0}^{N-1} \delta \boldsymbol{v}^{n} \cdot\left(h \mathrm{D}_{2} L\left(\boldsymbol{q}^{n}, \boldsymbol{v}^{n}\right)-h \boldsymbol{p}^{n+1}-h^{2} \sum_{k=1}^{m} \gamma_{k}^{n+1} \mathrm{D}^{2} g_{k}(\overline{\boldsymbol{q}}) \boldsymbol{M}^{-1} \boldsymbol{p}^{n+1}\right)=0
\end{aligned}
$$


along with

$$
\begin{aligned}
& \sum_{n=0}^{N-1} \delta \boldsymbol{\lambda}^{n+1} \cdot \boldsymbol{g}\left(\boldsymbol{q}^{n+1}\right)=0 \\
& \sum_{n=0}^{N-1} \delta \boldsymbol{\gamma}^{n+1} \cdot \boldsymbol{G}(\overline{\boldsymbol{q}}) \boldsymbol{M}^{-1} \boldsymbol{p}^{n+1}=0
\end{aligned}
$$

Applying an index shift in the second part of (40b) from $n+1$ to $n$ and taking into account the arbitrariness of all variations, we obtain the discrete EL equations

$$
\begin{aligned}
\boldsymbol{q}^{n+1}-\boldsymbol{q}^{n} & =h \boldsymbol{v}^{n}+h \boldsymbol{M}^{-1} \boldsymbol{G}(\overline{\boldsymbol{q}})^{\mathrm{T}} \boldsymbol{\gamma}^{n+1}, \\
\boldsymbol{p}^{n+1}-\boldsymbol{p}^{n} & =h \mathrm{D}_{1} L\left(\boldsymbol{q}^{n}, \boldsymbol{v}^{n}\right)-h \boldsymbol{G}\left(\boldsymbol{q}^{n}\right)^{\mathrm{T}} \boldsymbol{\lambda}^{n}-h \sum_{k=1}^{m} \gamma_{k}^{n+1} \mathrm{D}^{2} g_{k}(\overline{\boldsymbol{q}}) \boldsymbol{M}^{-1} \boldsymbol{p}^{n}, \\
\mathrm{D}_{2} L\left(\boldsymbol{q}^{n}, \boldsymbol{v}^{n}\right) & =\left(\boldsymbol{I}_{d \times d}+h \sum_{k=1}^{m} \gamma_{k}^{n+1} \mathrm{D}^{2} g_{k}(\overline{\boldsymbol{q}}) \boldsymbol{M}^{-1}\right) \boldsymbol{p}^{n+1}, \\
\boldsymbol{g}\left(\boldsymbol{q}^{n+1}\right) & =\mathbf{0}, \\
\boldsymbol{G}(\overline{\boldsymbol{q}}) \boldsymbol{M}^{-1} \boldsymbol{p}^{n+1} & =\mathbf{0},
\end{aligned}
$$

for $n=0, \ldots, N-1$. In total we have obtained a set of $(3 d+2 m)$ equations for the unknowns $\left(\boldsymbol{q}^{n+1}, \boldsymbol{p}^{n+1}, \boldsymbol{v}^{n}, \boldsymbol{\lambda}^{n}, \boldsymbol{\gamma}^{n+1}\right)$ in every time step. These are discrete counterparts of the continuous EL equations given in (28). It is advantageous that, due to the enhancement of the discrete action integral, the secondary constraints are now taken into account as well (cf. relation (41e)). Note that relation (41c) can be interpreted as the discrete fiber derivative of the Legendre transformation, which links velocity and momentum quantities. It is worth mentioning that scheme (41) can be regarded as generalization to constrained mechanical systems of the symplectic Euler method (see Hairer et al. [7] and Euler-B in Leimkuhler \& Reich [8]).

\subsection{Conservation Properties}

It is clear that the primary constraints are correctly captured in every time step by design (see relation (41d)). The secondary constraints are enforced in an intermediate sense (cf. (41e)).

Moreover, we can show that the integrator governed by (41) is symplectic. In order to demonstrate this, we calculate the differentials of (41a) to (41c). This yields

$$
\begin{aligned}
\mathrm{d} \boldsymbol{q}^{n+1}-\mathrm{d} \boldsymbol{q}^{n} & =h \mathrm{~d} \boldsymbol{v}^{n}+h \mathrm{~d}\left(\mathrm{D}_{2} \boldsymbol{g}^{\mathbf{v}}\left(\overline{\boldsymbol{q}}, \boldsymbol{p}^{n+1}\right)^{\mathrm{T}} \boldsymbol{\gamma}^{n+1}\right), \\
\mathrm{d} \boldsymbol{p}^{n+1}-\mathrm{d} \boldsymbol{p}^{n} & =h \mathrm{D}_{11}^{2} L\left(\boldsymbol{q}^{n}, \boldsymbol{v}^{n}\right) \mathrm{d} \boldsymbol{q}^{n}-h \mathrm{~d}\left(\boldsymbol{G}\left(\boldsymbol{q}^{n}\right)^{\mathrm{T}} \boldsymbol{\lambda}^{n}\right)-h \mathrm{~d}\left(\mathrm{D}_{1} \boldsymbol{g}^{\mathbf{v}}\left(\overline{\boldsymbol{q}}, \boldsymbol{p}^{n+1}\right)^{\mathrm{T}} \boldsymbol{\gamma}^{n+1}\right) \\
\mathrm{D}_{22}^{2} L\left(\boldsymbol{q}^{n}, \boldsymbol{v}^{n}\right) \mathrm{d} \boldsymbol{v}^{n} & =\mathrm{d} \boldsymbol{p}^{n+1}+h \mathrm{~d}\left(\mathrm{D}_{1} \boldsymbol{g}^{\mathbf{v}}\left(\overline{\boldsymbol{q}}, \boldsymbol{p}^{n+1}\right)^{\mathrm{T}} \boldsymbol{\gamma}^{n+1}\right) .
\end{aligned}
$$

where $\boldsymbol{g}^{\mathbf{v}}\left(\overline{\boldsymbol{q}}, \boldsymbol{p}^{n+1}\right)=\boldsymbol{G}(\overline{\boldsymbol{q}}) \boldsymbol{M}^{-1} \boldsymbol{p}^{n+1}$ has been introduced in analogy to the continuous case. Moreover, $\mathrm{D}_{12}^{2} L\left(\boldsymbol{q}^{n}, \boldsymbol{v}^{n}\right)=\mathrm{D}_{21}^{2} L\left(\boldsymbol{q}^{n}, \boldsymbol{v}^{n}\right)^{\mathrm{T}}=\mathbf{0}$ as been taken into account which is valid for Lagrangians of the form (3). The differential forms of the constraint equations (41d) and (41e) read

$$
\begin{aligned}
\mathrm{d} \boldsymbol{g}\left(\boldsymbol{q}^{n+1}\right) & =\boldsymbol{G}\left(\boldsymbol{q}^{n+1}\right) \mathrm{d} \boldsymbol{q}^{n+1}=\mathbf{0}, \\
\mathrm{d} \boldsymbol{g}^{\mathbf{v}}\left(\overline{\boldsymbol{q}}, \boldsymbol{p}^{n+1}\right) & =\mathrm{D}_{1} \boldsymbol{g}^{\mathbf{v}}\left(\overline{\boldsymbol{q}}, \boldsymbol{p}^{n+1}\right) \mathrm{d} \overline{\boldsymbol{q}}+\mathrm{D}_{2} \boldsymbol{g}^{\mathbf{v}}\left(\overline{\boldsymbol{q}}, \boldsymbol{p}^{n+1}\right) \mathrm{d} \boldsymbol{p}^{n+1}=\mathbf{0} .
\end{aligned}
$$

Now, making use of the skew-symmetry of the wedge product, property (17a), one can deduce that

$$
\mathrm{d} \boldsymbol{p}^{n+1} \wedge\left(\mathrm{d} \boldsymbol{q}^{n+1}-\mathrm{d} \boldsymbol{q}^{n}\right)+\left(\mathrm{d} \boldsymbol{p}^{n+1}-\mathrm{d} \boldsymbol{p}^{n}\right) \wedge \mathrm{d} \boldsymbol{q}^{n}=\mathrm{d} \boldsymbol{q}^{n} \wedge \mathrm{d} \boldsymbol{p}^{n}-\mathrm{d} \boldsymbol{q}^{n+1} \wedge \mathrm{d} \boldsymbol{p}^{n+1} .
$$

Substituting from (42a) and (42b) into the last equation, we obtain

$$
\begin{aligned}
\mathrm{d} \boldsymbol{q}^{n} & \wedge \mathrm{d} \boldsymbol{p}^{n}-\mathrm{d} \boldsymbol{q}^{n+1} \wedge \mathrm{d} \boldsymbol{p}^{n+1}=\mathrm{d} \boldsymbol{p}^{n+1} \wedge h \mathrm{~d} \boldsymbol{v}^{n}+h \mathrm{~d} \boldsymbol{p}^{n+1} \wedge \mathrm{d}\left(\mathrm{D}_{2} \boldsymbol{g}^{\mathbf{v}}\left(\overline{\boldsymbol{q}}, \boldsymbol{p}^{n+1}\right)^{\mathrm{T}} \boldsymbol{\gamma}^{n+1}\right) \\
& +h \mathrm{D}_{11}^{2} L\left(\boldsymbol{q}^{n}, \boldsymbol{v}^{n}\right) \mathrm{d} \boldsymbol{q}^{n} \wedge \mathrm{d} \boldsymbol{q}^{n}-h \mathrm{~d}\left(\boldsymbol{G}\left(\boldsymbol{q}^{n}\right)^{\mathrm{T}} \boldsymbol{\lambda}^{n}\right) \wedge \mathrm{d} \boldsymbol{q}^{n}-h \mathrm{~d}\left(\sum_{k=1}^{m} \gamma_{k}^{n+1} \mathrm{D}_{1} \boldsymbol{g}_{k}^{\mathbf{v}}\left(\overline{\boldsymbol{q}}, \boldsymbol{p}^{n+1}\right)\right) \wedge \mathrm{d} \boldsymbol{q}^{n} .
\end{aligned}
$$


We next insert $\mathrm{d} \boldsymbol{p}^{n+1}$ from (42c) into the first term on the right-hand side of (45). Moreover, the third term on the right-hand side of (45) vanishes due to property (17d) of the wedge product. The fourth one vanishes in analogy to relation (37). Consequently, we obtain

$$
\begin{aligned}
\mathrm{d} \boldsymbol{q}^{n} & \wedge \mathrm{d} \boldsymbol{p}^{n}-\mathrm{d} \boldsymbol{q}^{n+1} \wedge \mathrm{d} \boldsymbol{p}^{n+1}=\left(\mathrm{D}_{22}^{2} L\left(\boldsymbol{q}^{n} \boldsymbol{v}^{n}\right) \mathrm{d} \boldsymbol{v}^{n}-h \mathrm{~d}\left(\sum_{k=1}^{m} \gamma_{k}^{n+1} \mathrm{D}_{1} \boldsymbol{g}_{k}^{\mathbf{v}}\left(\overline{\boldsymbol{q}}, \boldsymbol{p}^{n+1}\right)\right)\right) \wedge h \mathrm{~d} \boldsymbol{v}^{n} \\
& +h \mathrm{~d} \boldsymbol{p}^{n+1} \wedge \mathrm{d}\left(\mathrm{D}_{2} \boldsymbol{g}^{\mathbf{v}}\left(\overline{\boldsymbol{q}}, \boldsymbol{p}^{n+1}\right)^{\mathrm{T}} \boldsymbol{\gamma}^{n+1}\right)-h \mathrm{~d}\left(\sum_{k=1}^{m} \gamma_{k}^{n+1} \mathrm{D}_{1} \boldsymbol{g}_{k}^{\mathbf{v}}\left(\overline{\boldsymbol{q}}, \boldsymbol{p}^{n+1}\right)\right) \wedge \mathrm{d} \boldsymbol{q}^{n}
\end{aligned}
$$

The symmetric matrix multiplication property of the wedge product (17d) can be used once more to cancel the first term on the right-hand side of (46). Moreover, it is possible to collect the second and fourth term, yielding

$$
\begin{aligned}
\mathrm{d} \boldsymbol{q}^{n} & \wedge \mathrm{d} \boldsymbol{p}^{n}-\mathrm{d} \boldsymbol{q}^{n+1} \wedge \mathrm{d} \boldsymbol{p}^{n+1}= \\
& -h \mathrm{~d}\left(\sum_{k=1}^{m} \gamma_{k}^{n+1} \mathrm{D}_{1} \boldsymbol{g}_{k}^{\mathbf{v}}\left(\overline{\boldsymbol{q}}, \boldsymbol{p}^{n+1}\right)\right) \wedge \mathrm{d} \overline{\boldsymbol{q}}+h \mathrm{~d} \boldsymbol{p}^{n+1} \wedge \mathrm{d}\left(\mathrm{D}_{2} \boldsymbol{g}^{\mathbf{v}}\left(\overline{\boldsymbol{q}}, \boldsymbol{p}^{n+1}\right)^{\mathrm{T}} \boldsymbol{\gamma}^{n+1}\right)
\end{aligned}
$$

Executing the remaining differentials leads to the expression

$$
\begin{aligned}
\mathrm{d} \boldsymbol{q}^{n} & \wedge \mathrm{d} \boldsymbol{p}^{n}-\mathrm{d} \boldsymbol{q}^{n+1} \wedge \mathrm{d} \boldsymbol{p}^{n+1}= \\
& \left(\mathrm{D}_{1} \boldsymbol{g}^{\mathbf{v}}\left(\overline{\boldsymbol{q}}, \boldsymbol{p}^{n+1}\right) \mathrm{d} \overline{\boldsymbol{q}}\right) \wedge h \mathrm{~d} \boldsymbol{\gamma}^{n+1}+\left(\mathrm{D}_{2} \boldsymbol{g}^{\mathbf{v}}\left(\overline{\boldsymbol{q}}, \boldsymbol{p}^{n+1}\right) \mathrm{d} \boldsymbol{p}^{n+1}\right) \wedge h \mathrm{~d} \boldsymbol{\gamma}^{n+1} \\
& -h \sum_{k=1}^{m} \gamma_{k}^{n+1} \mathrm{D}_{12}^{2} g_{k}^{\mathbf{v}}\left(\overline{\boldsymbol{q}}, \boldsymbol{p}^{n+1}\right) \mathrm{d} \boldsymbol{p}^{n+1} \wedge \mathrm{d} \overline{\boldsymbol{q}}+h \mathrm{~d} \boldsymbol{p}^{n+1} \wedge \sum_{k=1}^{m} \gamma_{k}^{n+1} \mathrm{D}_{21}^{2} g_{k}^{\mathbf{v}}\left(\overline{\boldsymbol{q}}, \boldsymbol{p}^{n+1}\right) \mathrm{d} \overline{\boldsymbol{q}}
\end{aligned}
$$

In analogy to the proof given in Sect. 3.3, terms including $\mathrm{D}_{11}^{2} g_{k}^{\mathbf{v}}$ and $\mathrm{D}_{22}^{2} g_{k}^{\mathbf{v}}$, respectively, cancel due to their symmetry. It becomes obvious that the last two terms on the right-hand side of (48) cancel each other out due to $(17 \mathrm{c})$ since $\left(\mathrm{D}_{12}^{2} g_{k}^{\mathbf{v}}\right)^{\mathrm{T}}=\mathrm{D}_{12}^{2} g_{k}^{\mathrm{v}}$. The first two terms on the right-hand side of (48) can be collected such that (43b) can be taken into account. Eventually, the whole expression on the right-hand side of (48) vanishes and we obtain

$$
\mathrm{d} \boldsymbol{q}^{n} \wedge \mathrm{d} \boldsymbol{p}^{n}=\mathrm{d} \boldsymbol{q}^{n+1} \wedge \mathrm{d} \boldsymbol{p}^{n+1},
$$

which shows that the present scheme is indeed symplectic.

\section{NUMERICAL EXAMPLE}

The objective of this subsection is to analyze the behavior of the previously derived symplectic method by means of a numerical example. We investigate the motion of a rigid body according to a director framework which has already been used by Betsch \& Steinmann [18], Krenk \& Nielsen [19] and many others. This formulation describes rigidity using the orthonormality condition of three directors $\left\{\boldsymbol{d}_{i}\right\}$ positioned in the center of mass $\boldsymbol{\varphi}$. Consequently, every point is uniquely defined by its material coordinates $X_{i}$ with respect to the director frame's origin such that we are able to express the spatial placement as a function of the material coordinates and time such that

$$
\boldsymbol{x}(\boldsymbol{X}, t)=\boldsymbol{\varphi}(t)+X_{i} \boldsymbol{d}_{i}(t)
$$

where the summation convention applies. Eventually, it is possible to describe the motion of the rigid body by $n=3+9=12$ redundant coordinates accumulated in the coordinate vector

$$
q=\left[\begin{array}{c}
\varphi \\
d_{1} \\
d_{2} \\
d_{3}
\end{array}\right]
$$




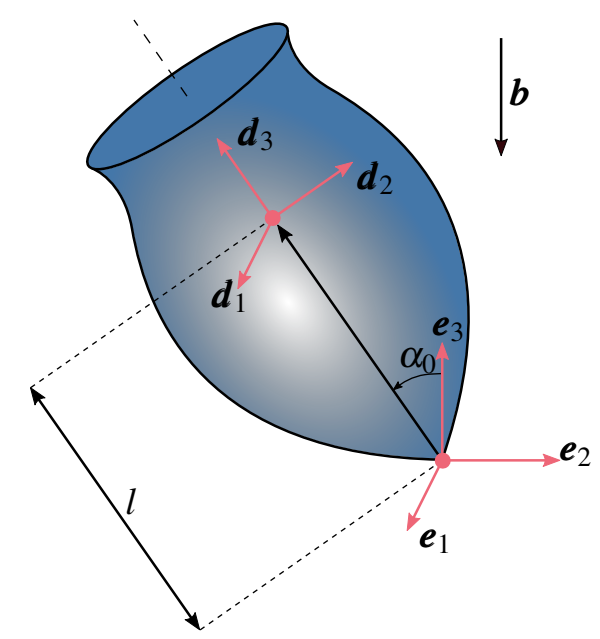

Figure 2. Initial configuration of the gyroscopic top

This framework consequently allows for a representation of the system's Lagrangian in the standard fashion (3) with the constant and diagonal mass matrix

$$
\boldsymbol{M}=\left[\begin{array}{cccc}
m \boldsymbol{I} & \mathbf{0} & \mathbf{0} & \mathbf{0} \\
\mathbf{0} & E_{1} \boldsymbol{I} & \mathbf{0} & \mathbf{0} \\
\mathbf{0} & \mathbf{0} & E_{2} \boldsymbol{I} & \mathbf{0} \\
\mathbf{0} & \mathbf{0} & \mathbf{0} & E_{3} \boldsymbol{I}
\end{array}\right],
$$

where principle values of the Euler tensor can be computed with the principal moments of inertia as $E_{i}=1 / 2\left(J_{j}+J_{k}-J_{i}\right)$ for even permutations of the indices $(i, j, k)$. The primary constraints enforce the directors to stay orthonormal for all times due to the rigidity of the body, viz.

$$
\boldsymbol{g}(\boldsymbol{q})=\tilde{\boldsymbol{g}}\left(\left\{\boldsymbol{d}_{\mathrm{i}}\right\}\right)=\left[\begin{array}{c}
\frac{1}{2}\left(\boldsymbol{d}_{1} \cdot \boldsymbol{d}_{1}-1\right) \\
\frac{1}{2}\left(\boldsymbol{d}_{2} \cdot \boldsymbol{d}_{2}-1\right) \\
\frac{1}{2}\left(\boldsymbol{d}_{3} \cdot \boldsymbol{d}_{3}-1\right) \\
\boldsymbol{d}_{1} \cdot \boldsymbol{d}_{2} \\
\boldsymbol{d}_{1} \cdot \boldsymbol{d}_{3} \\
\boldsymbol{d}_{2} \cdot \boldsymbol{d}_{3}
\end{array}\right]=\mathbf{0}
$$

Specifically in this example, a gyroscopic top, as depicted in Fig. 2, has been investigated for a total simulation time of $T=2 \mathrm{~s}$ with a time step size of $h=0.002 \mathrm{~s}$ computed with the symplectic variational integrator from Sect. 4.

The total mass of the top amounts to $m=0.7069 \mathrm{~kg}$ and the momenta of inertia read $J_{1}=J_{2}=$ $J_{3}=5.3014 \cdot 10^{-4} \mathrm{~kg} \mathrm{~m}^{2}$ which corresponds to a cylinder with mass density $\rho=2700 \mathrm{~kg} / \mathrm{m}^{3}$, height $a=0.1 \mathrm{~m}$, top radius $r=a / 2$ and a location of the center of mass along the symmetry axis $l=3 / 4 a$. In this case, the momenta of inertia can be computed via

$$
J_{1}=J_{2}=\frac{3}{80} m\left(4 r^{2}+a^{2}\right), \quad J_{3}=\frac{3}{10} m r^{2},
$$

and the total mass is given by $m=\frac{1}{3} \rho \pi r^{2} a$. Gravitation acts in the negative $\boldsymbol{e}_{3}$-direction with $\boldsymbol{b}=\left[0,0,-9.81 \mathrm{~m} / \mathrm{s}^{2}\right]^{\mathrm{T}}$ such that the potential energy of the system only depends on the position of the center of mass $\varphi$, viz.

$$
V(\boldsymbol{q})=\hat{V}(\boldsymbol{\varphi})=-m \boldsymbol{b} \cdot \boldsymbol{\varphi} .
$$

It is crucially important that the top is subject to an additional constraint

$$
g_{\mathrm{cm}}(\boldsymbol{q})=\boldsymbol{\varphi}-\boldsymbol{l d}_{3}=0,
$$


which fixes the tip of the gyroscopic top to the origin of $\left\{\boldsymbol{e}_{i}\right\}$ by enforcing that the center of mass is located on the axis of symmetry with a distance of $l$ to the origin. The initial nutation angle is $\alpha_{0}=\pi / 3$ and the gyroscopic top is subject to an initial angular velocity vector

$$
\boldsymbol{\omega}_{0}=\omega_{\mathrm{p}} \boldsymbol{e}_{3}+\omega_{\mathrm{s}} \boldsymbol{d}_{3},
$$

where the initial precession rate is chosen as $\omega_{p}=10 \mathrm{~s}^{-1}$ and the initial spin rate for the case of steady precession can be computed via the relation

$$
\omega_{\mathrm{s}}=\frac{m g l}{J_{3} \omega_{\mathrm{p}}}+\frac{J_{1}+m l^{2}-J_{3}}{J_{3}} \omega_{p} \cos \left(\alpha_{0}\right)
$$

(cf. p. 221 in Goldstein [20]), which amounts to $\omega_{\mathrm{s}}=135.6 \mathrm{~s}^{-1}$ for the present case. Note that $g=9.81 \mathrm{~m} / \mathrm{s}^{2}$ denotes the magnitude of gravitational acceleration here. The transformation from the global $\boldsymbol{e}_{i}$ coordinate system to the initially inclined system can easily be done by the use of a rotation matrix $\boldsymbol{R}_{0} \in S O(3)$ with the well-known property $\boldsymbol{R}_{0}^{\mathrm{T}}=\boldsymbol{R}_{0}^{-1}$. For the present case this reads

$$
\boldsymbol{R}_{0}=\left[\begin{array}{ccc}
1 & 0 & 0 \\
0 & \cos \left(\alpha_{0}\right) & -\sin \left(\alpha_{0}\right) \\
0 & \sin \left(\alpha_{0}\right) & \cos \left(\alpha_{0}\right)
\end{array}\right],
$$

which prescribes a rotation about the $\boldsymbol{e}_{1}$-axis with the angle $\alpha_{0}$ as can be seen in Fig. 2. Thus, the initial velocities of the center of mass and the directors can be computed by taking the cross product with the initial configuration

$$
\dot{\boldsymbol{\varphi}}(t=0)=\boldsymbol{\omega}_{0} \times \boldsymbol{\varphi}(t=0), \quad \dot{\boldsymbol{d}}_{\mathrm{i}}(t=0)=\boldsymbol{\omega}_{0} \times \boldsymbol{d}_{\mathrm{i}}(t=0),
$$

where $\boldsymbol{\omega}_{0}$ can be transformed to the global coordinate system first with the aid of $\boldsymbol{R}_{0}$ to simplify the computation. The Newton's method's tolerance has been set to $\varepsilon_{\text {tol }}=10^{-9}$. The computation has been performed using the metis code, which is available at [21].

By ensuring the condition for a steady precession (58) the center of mass is rotating on a constant height $\varphi_{3}$ around the vertical axis since gravitational forces and restabilizing effects due to the rotation are in an equilibrium. The horizontal coordinate of the center of mass $\varphi_{3}$ can thus be regarded as an analytical reference to the solutions. The results given by the symplectic integration scheme oscillates around this analytical solution, as can be seen in Fig. 10.

The evolution of the energetic quantities $T, V$, and $H$ is shown in Fig. 3. The total energy of the system is not conserved identically along the solutions of this integration scheme as it can be seen in Fig. 5 which displays the increments in $H$ from one time step to another. However, as it is typical for symplectic methods (cf. Fig. 16 in Lew \& Mata [10]), $H(t)$ oscillates around its true value and the energy error remains stable. Furthermore, as the gyroscopic top is subject to external forces acting in the $\boldsymbol{e}_{3}$-direction, the symmetry of the system reduces to a conservation of the angular

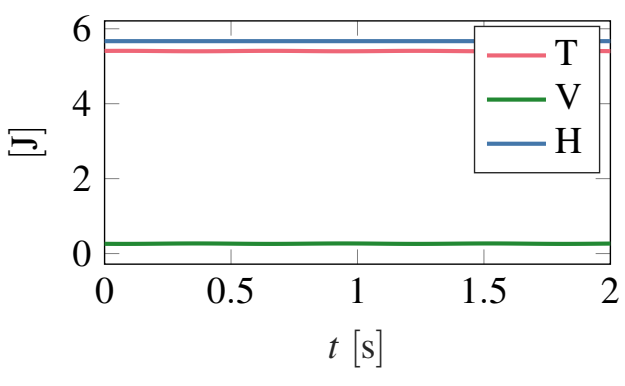

Figure 3. Energy quantities

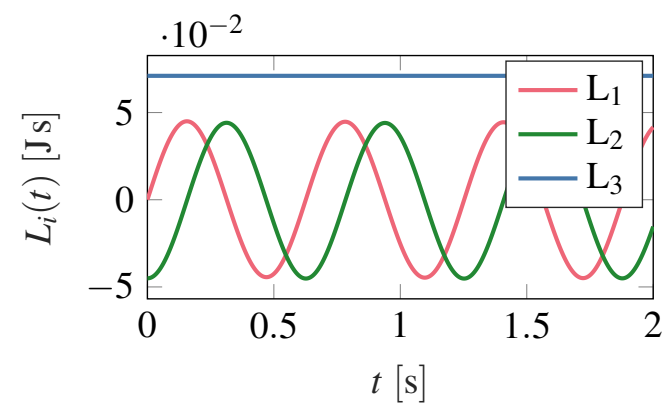

Figure 4. Angular momentum 


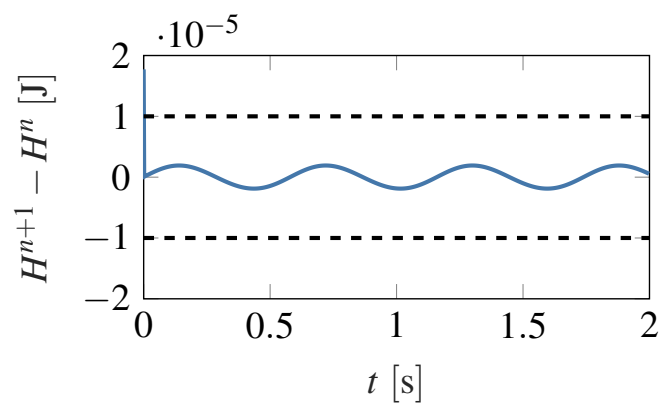

Figure 5. Hamiltonian difference

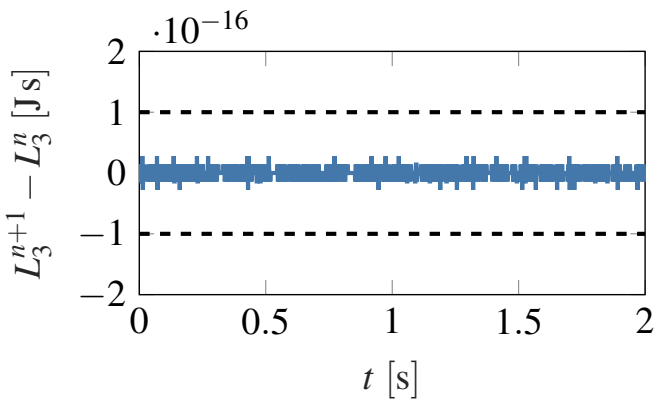

Figure 6. Angular momentum difference

momentum about the $\boldsymbol{e}_{3}$-axis such that $L_{3}=$ constant. This is correctly captured by the symplectic method and can be seen in Fig. 4. Differences in $L_{3}$ from one point in time to another are close to computer precision (cf. Fig. 6).

By design, the constraints on configuration level are identically fulfilled which can be observed in Fig. 7. In contrast to that, in each time step the secondary constraints are merely enforced in an intermediate configuration leading to the results depicted in Fig. 8.

We can moreover analyze the h-convergence of the symplectic variational integrator. Therefore we have investigated the relative error in the vertical coordinate of the center of mass, which is supposed to remain constantly $\phi_{3, \text { ana }}=0.0375 \mathrm{~m}$, after a total simulation time of $t=0.001 \mathrm{~s}$ for various time step sizes. In Fig. 9 we display the relative error

$$
e=\frac{\left|\varphi_{3}(t=0.001 \mathrm{~s})-\varphi_{3, \text { ana }}\right|}{\varphi_{3, \text { ana }}}
$$

for the different time step sizes $h$. It becomes visible that the present method is first order accurate.

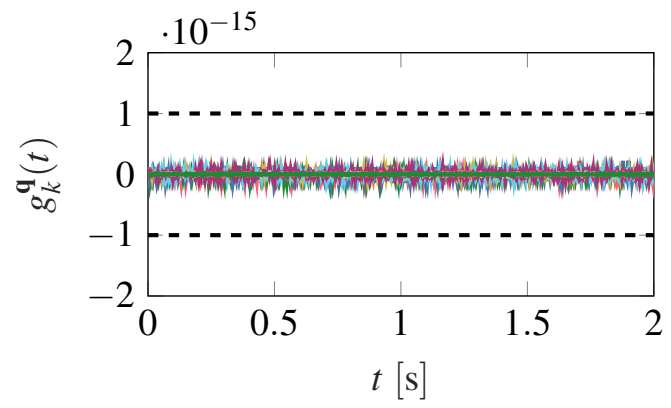

Figure 7. Constraints on configuration level

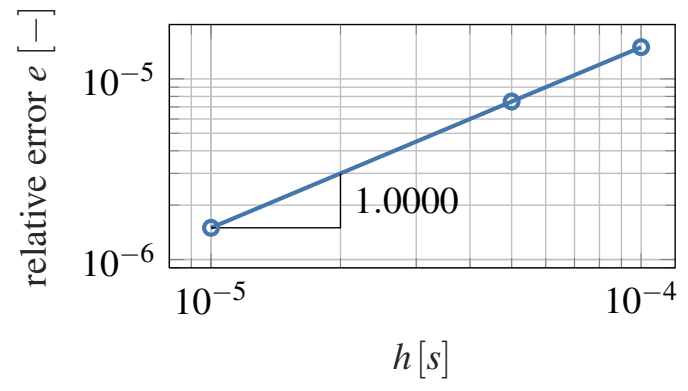

Figure 9. h-convergence of relative error in $\boldsymbol{q}$

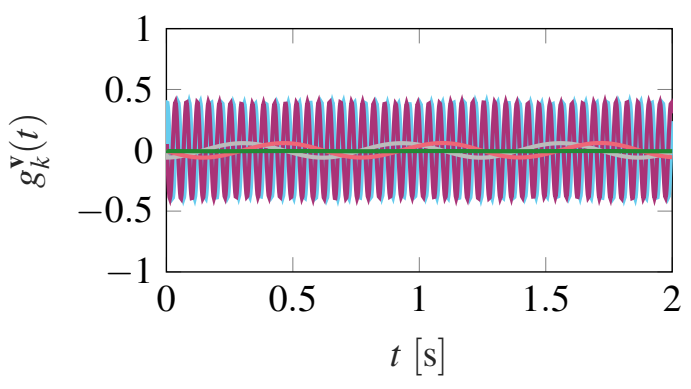

Figure 8. Constraints on velocity level

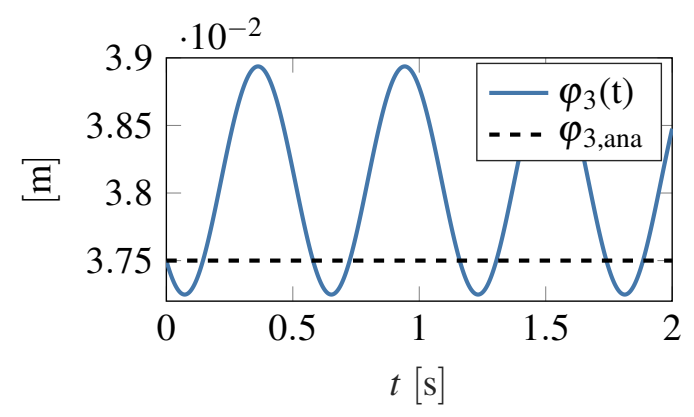

Figure 10. Vertical coordinate of center of mass 


\section{CONCLUSION AND OUTLOOK}

In this work, a new variational principle for the analysis of constrained dynamics has been proposed. The underlying functional takes account of both primary and secondary constraints. Due to its mixed character with independent position, velocity and momentum quantities, it generalizes Livens principle [1] and thus unites Lagrangian and Hamiltonian viewpoints. By coupling constraints on position and velocity level into the equations we have obtained a set of DAEs which can be regarded as an extension of the well-known GGL stabilization [15]. Contrary to the original formulation, however, the emanating equations of motion have Hamiltonian structure.

The novel GGL functional gives rise to DAEs with differentiation index 2 and thus circumvents the numerical problems of the standard index-3 DAEs pertaining to mechanical systems subject to holonomic constraints. We could show, that the formulation is symplectic and has Hamiltonian structure. The conservation principles of constrained dynamics can be carried over to the novel augmented formulation. We have demonstrated that, in analogy to the classical GGL formulation [15], in the time-continuous case the additional Lagrange multipliers need to vanish. However, in contrast to the original GGL formulation, this property is not required to retain the conservation of the Hamiltonian and the symplectic structure.

Based on the newly proposed variational principle, we have succesfully derived a new first-order variational integrator. This integrator satisfies the primary constraints and is capable to conserve the angular momentum of the system. We could show that the method is symplectic which is a typical property of variational integrators (cf. Marsden \& West [11]). The secondary constraints have been taken into account in an intermediate sense.

The novel variational framework represents a promising basis for the construction of structurepreserving integration schemes. The method which has been deduced throughout this work can thus be seen as a starting point for further developments. In particular, due to the close relationship of the GGL principle to optimal control, previously developed direct methods based on the philosophy "first discretize then optimize" (see, for example, Betsch \& Becker [22]) can be used to obtain higher-order variational integrators for constrained mechanical systems. These integrators are symplectic by design. Furthermore, slight modifications can be applied to obtain energymomentum consistent integrators which represent another important class of structure-preserving time-stepping schemes.

\section{ACKNOWLEDGEMENTS}

This work was funded by the Deutsche Forschungsgemeinschaft (DFG, German Research Foundation) - project number 227928419 (BE 2285/9-3). This support is gratefully acknowledged.

\section{REFERENCES}

[1] Livens, G.H.: On Hamilton's principle and the modified function in analytical dynamics. Proceedings of the Royal Society Edinburgh 39(IX) (1919) 113-119

[2] Bou-Rabee, N., Marsden, J.E.: Hamilton-Pontryagin Integrators on Lie Groups Part I: Introduction and Structure-Preserving Properties. Foundations of Computational Mathematics 9(2) (2009) 197-219

[3] Yoshimura, H., Marsden, J.E.: Dirac structures in Lagrangian mechanics Part II: Variational structures. Journal of Geometry and Physics 57(1) (2006) 209-250

[4] Holm, D.D.: Geometric Mechanics: Part II: Rotating, Translating and Rolling. 2 edn. Imperial College Press (2011)

[5] Pontryagin, L., Boltayanskii, V., Gamkrelidze, R., Mishchenko, E.: The mathematical theory of optimal processes. Wiley (1962) 
[6] Kunkel, P., Mehrmann, V.: Differential-algebraic equations: analysis and numerical solution. EMS textbooks in mathematics. European Mathematical Society (2006)

[7] Hairer, E., Lubich, C., Wanner, G.: Geometric numerical integration: structure-preserving algorithms for ordinary differential equations. Springer (2006)

[8] Leimkuhler, B., Reich, S.: Simulating Hamiltonian Dynamics. Cambridge Monographs on Applied and Computational Mathematics. Cambridge University Press (2005)

[9] De Vogelaere, R.: Methods of Integration which Preserve the Contact Transformation Property of the Hamilton Equations. Technical report (University of Notre Dame. Dept. of Mathematics) (1956)

[10] Lew, A.J., Mata, P.: A Brief Introduction to Variational Integrators. In Betsch, P., ed.: Structure-preserving Integrators in Nonlinear Structural Dynamics and Flexible Multibody Dynamics. Volume 565. Springer (2016) 201-291

[11] Marsden, J.E., West, M.: Discrete mechanics and variational integrators. Acta Numerica 10 (2001) 357-514

[12] Maeda, S.: Lagrangian formulation of discrete systems and concept of difference space. Math. Japon 27 (1982) 345-356

[13] Leyendecker, S., Marsden, J., Ortiz, M.: Variational integrators for constrained dynamical systems. ZAMM - Journal of Applied Mathematics and Mechanics / Zeitschrift für Angewandte Mathematik und Mechanik 88(9) (2008) 677-708

[14] Yoshimura, H.: A Geometric Method of Constraint Stabilization for Holonomic Mechanical Systems. Proceedings of ACMD06 A00712 (2006)

[15] Gear, C., Leimkuhler, B., Gupta, G.: Automatic integration of Euler-Lagrange equations with constraints. Journal of Computational and Applied Mathematics 12-13 (1985) 77-90

[16] Pars, L.A.: A Treatise on Analytical Dynamics. The Mathematical Gazette 50(372) (1966) 226-227

[17] Washizu, K.: Variational Methods in Elasticity and Plasticity. Pergamon Press (1975)

[18] Betsch, P., Steinmann, P.: Constrained integration of rigid body dynamics. Computer Methods in Applied Mechanics and Engineering 191(3-5) (2001) 467-488

[19] Krenk, S., Nielsen, M.B.: Conservative rigid body dynamics by convected base vectors with implicit constraints. Computer Methods in Applied Mechanics and Engineering 269 (2014) 437-453

[20] Goldstein, H.: Classical mechanics. 2 edn. Addison-Wesley Pub. Co. (1980)

[21] Kinon, P.L.: metis: computing constrained dynamical systems (github repository). https: //github.com/philipplk/metis (2021)

[22] Betsch, P., Becker, C.: Conservation of generalized momentum maps in mechanical optimal control problems with symmetry. International Journal for Numerical Methods in Engineering 111(2) (2017) 144-175 